International Journal of Computer Science \& Information Technology (IJCSIT) Vol 3, No 4, August 2011

\title{
NOVEL APPROACH FOR SPEECH RECOGNITION BY USING SELF - ORGANIZED MAPS
}

\author{
DR.R.L.K.VENKATESWARLU ${ }^{1}$, DR. R. VASANTHA KUMARI ${ }^{2}$ AND \\ A.K.V.NAGAYYA ${ }^{3}$ \\ ${ }^{1}$ Professor and Head, Department of Information Technology \\ Sasi Institute of Technology and Engineering, Tadepalligudem, INDIA. \\ rlk.maths@gmail.com \\ ${ }^{2}$ Principal \\ Perunthalaivar Kamarajar Arts College,PUDUCHERRY 605107 \\ Vasuntharalegmail.com \\ ${ }^{3}$ Lecturer, Department of Information Technology \\ Sasi Institute of Technology \& Engineering, Tadepalligudem, INDIA. \\ akvn1983@gmail.com
}

\begin{abstract}
The method of self-organizing maps (SOM) is a method of exploratory data analysis used for clustering and projecting multi-dimensional data into a lower-dimensional space to reveal hidden structure of the data. The Self-Organizing Feature Maps (SOFMs) [11] is a class of neural networks capable of recognizing the main features of the data they are trained on. There is extensive literature on its biological and mathematical concepts and even more on its implementation in a variety of areas including medicine, finance, chaos and data mining in general [4,2]. The aim of this research is to implement a self-organizing neural network based technique for speech recognition. The Mean-SOM performance for the feature Intensity is obtained maximum as $98.17 \%$. The Median-SOM performance for the feature Intensity is obtained maximum as $98.54 \%$.
\end{abstract}

KEYWORDS: Self-organized map, Artificial Neural Networks, Feature, Mean-SOM performance, Median-SOM performance, LPCC, MFCC, Pitch, Intensity, Hits, Cycles, Iterations.

\section{INTRODUCTION}

The SOMs are usually defined in metric vector spaces. In SOMs of symbol strings or other nonvectorial representations, the relative locations of the images of the strings on the SOM ought to reflect $[10,8]$. The self-organizing feature map (SOFM) is primarily used to map highdimensional data into low-dimensional spaces for pattern classification applications [1]. Some well known theoretical results concerning the universal approximation property of MLP neural networks with one hidden layer have shown that for any function $\mathrm{f}$ from $[0,1] \mathrm{n}$ to $\mathrm{R}$, only the output layer weights depend on $\mathrm{f}$. This result is used to propose a network architecture called the predictive Kohonen map allowing to design a new speech features extractor. The SOMs are usually defined in metric vector spaces [7]. 


\subsection{SOM Network Architecture}

The hidden layer of an ANN is one of the most complex parts to design in an artificial neural network. This section proposes a Supervised SOM Based Architecture, which consists of Input layer, Competitive layer and Output layer.

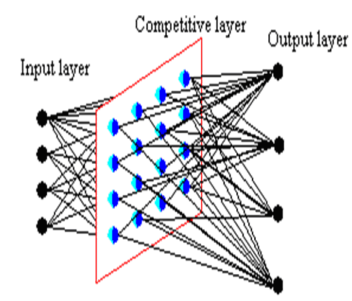

\section{Input Layer}

Fig.1 SOM Network Architecture

Accepts multidimensional input pattern from the environment. An input pattern is represented by a vector. Each neurode in the input layer represents one dimension of the input pattern. An input neurode distributes its assigned element of the input vector to the competitive layer.

\section{Competitive layer}

Each neurode in the competitive layer receives a sum of weighted inputs from the input layer. Every neurode in the competitive layer is associated with a collection of other neurodes which make up its 'neighbourhood'. We can organize Competitive layer on any dimension. Upon receipt of a given input, some of the neurodes will be sufficiently excited to fire. This event can have either an inhibitory, or an excitatory effect on its neighborhood. The model has been copied from biological systems, and is known as 'on-center, off-surround' architecture, also known as lateral feedback / inhibition.

\section{Output layer}

Organization of the output layer is application-dependent. Strictly speaking, not necessary for proper functioning of a Kohonen network. The "output" of the network is the way we choose to view the interconnections between nodes in the competitive layer. If nodes are arranged along a single dimension, output can be seen as a continuum:

A self-organizing map (newsom) consists of a competitive layer which can classify a dataset of vectors with any number of dimensions into as many classes as the layer has neurons. The neurons are arranged in a 2D topology, which allows the layer to form a representation of the distribution and a two-dimensional approximation of the topology of the dataset. The network is trained with the SOM batch algorithm.

\subsection{SELF ORGANIZATION MAP}

The principal goal of the SOM is to transform an incoming signal pattern of arbitrary dimension into one or two dimensional discrete map, and to perform this transformation adaptively in a topologically order fashion.

There are three essential processes involved in the formation of the SOM.

Competition: For each input pattern, the neurons in the network compute their respective values of a discriminant function. This discriminant function provides the basis for competition among 
the neurons. The particular neuron with the largest value of discriminant function is declared winner of the competition.

Cooperation: The winning neuron determines the spatial location of a topological neighborhood excited neurons, thereby providing the basis for cooperation among such neighborhood neurons.

Synaptic Adaptation: This last mechanism enables the excited neurons to increase their individual values of the discriminant function in relation to the input pattern through suitable adjustments applied to their synaptic weights. The adjustments made are such that the response of the winning neuron to the subsequent application of a similar input pattern is enhanced.

Self-organizing feature maps (SOFM) learn to classify input vectors according to how they are grouped in the input space. They differ from competitive layers in that neighboring neurons in the self-organizing map learn to recognize neighboring sections of the input space. Thus, selforganizing maps learn both the distribution and topology of the input vectors they are trained on.

\subsection{SOM ALGORITHM}

Kohonen's Self Organizing Feature Map: The self organizing feature map developed by Kohonen which groups the input data into clusters. Self-Organizing Map (SOM), with its variants, is the most popular artificial neural network algorithm in the unsupervised learning category [3]. The Kohonen learning algorithm is controlled by two learning parameters, which have to be chosen empirically because there exists neither rules nor a method for their calculation [5].

The Kohonen algorithm is

Step1. Initialization:

Set neighborhood parameter $h$ and learning rate $\alpha$.

Initialize the weight vectors: $\mathrm{w} 1, \mathrm{w} 2, \mathrm{w} 3, \ldots \ldots, \mathrm{wm}$ of $\mathrm{m}$ computing units are selected at a random.

Step2. Sampling: Draw sample input vector $\mathrm{X}$ from input space.

Step3. Matching: Find the best-matching for winning neuron winner $=I(X)=$ minimum of $d j$.

Where $\mathrm{dj}$ is the squared Euclidean distance.

$d_{j}=\sum\left(x_{i}-w_{i j}\right)^{2} i=1$ to $m$ and $j=1$ to $n$

Euclidean distance square: Generally Euclidean norm length of an n-tuple vector $\mathrm{X}$ is denoted as $\|X\|_{\text {and }}\|X\|=\left(X^{T} X\right)^{1 / 2}$

Note that the Euclidean norm can be computed by using matrix multiplication. Indeed, for an ncomponent vector $\mathrm{X}$ can be written in the form 
International Journal of Computer Science \& Information Technology (IJCSIT) Vol 3, No 4, August 2011

$\|X\|=\left(\left[\begin{array}{lll}x_{1} & x_{2} & \ldots \ldots \ldots x_{n}\end{array}\right]\left(\begin{array}{c}x_{1} \\ x_{2} \\ \vdots \\ x_{n}\end{array}\right)^{1 / 2}=\left[\sum_{i=1}^{n} x_{i}^{2}\right]^{1 / 2}\right.$

Similarly we can write

$\left\|X_{i}-W_{i j}\right\|=\left[\sum_{i=1}^{n}\left\|x_{i}-w_{i j}\right\|^{2}\right]^{1 / 2}$

Therefore square of the Euclidean norm $=\left[\sum_{i=1}^{n}\left\|x_{i}-w_{i j}\right\|^{2}\right]$

Step4. Updating: Update the weights which are connected with the winner.

$w_{i j}(k+1)=w_{i j}(k)+\alpha \cdot h\left[x_{i}-w_{i j}(k)\right]$

or, $w_{i j}($ new $)=w_{i j}($ old $)+\alpha \cdot h\left[x_{i}-w_{i j}(\right.$ old $\left.)\right]$

Where $h$ is the neighborhood of the winning neuron $\mathrm{I}(\mathrm{X})$.

Which applied to all the neurons in the lattice that lie inside the topological neighborhood $\mathrm{h}$ of winning neuron $\mathrm{i}$.

Change the learning rate.

Reduce the radius.

Step5. Continuation: Return to step-2 until some changes in the feature map are observed. $w_{i j}(k+1)=w_{i j}(k)+\alpha \cdot h\left[x_{i}-w_{i j}(k)\right]$

\section{SYSTEM CONCEPT}

\subsection{Dataset}

Speech dictation of the word "Jaundice" is given and spelt as "Jaundice", "Zaundice", "Zaundis", "Jaundis", Johndis", Johndice", "Jhondis", "Jaandis", "Zaundees", Jaundees", "Jandice", "Jowndis" depending on the understanding capabilities (of the speakers). These words are then pronounced in a quite environment and uttered by five speakers (3Male, 2Female).

\subsection{Preprocessing}

The speech signals are recorded in a low noise environment with good quality recording equipment. The signals are samples at $11 \mathrm{kHz}$. Reasonable results can be achieved in isolated word recognition when the input data is surrounded by silence.

\subsection{Sampling Rate}

150 samples are chosen with sampling rate $11 \mathrm{kHz}$, which is adequate to represent all speech sounds.

\subsection{Windowing}

Inorder to avoid discontinuties at the end of speech segments the signal should be tapered to zero or near zero and hence reduce the mismatch. To the given 12 Mel-Frequency coefficients, and for time 0.005 seconds, a window length of 0.015 is selected by the Praat Object software tool.

\subsection{Features Extraction}

\subsubsection{Linear Predictive Cepstral Coefficients}

The goal of feature extraction is to represent speech signal by a finite number of measures of the signal. This is because the entirety of the information in the acoustic signal is too much to 
process, and not all of the information is relevant for specific tasks. In present Speech Recognition systems, the approach of feature extraction has generally been to find a representation that is relatively stable for different examples of the same speech sound, despite differences in the speaker or various environmental characteristics, while keeping the part that represents the message in the speech signal relatively intact.

Linear predictive coding (LPC) is a tool used mostly in audio signal processing and speech processing for representing the spectral envelope of a digital signal of speech in compressed form, using the information of a linear predictive mode. It is one of the most powerful speech analysis techniques, and one of the most useful methods for encoding good quality speech at a low bit rate and provides extremely accurate estimates of speech parameters.

LPC analyzes the speech signal by estimating the formats, removing their effects from the speech signal, and estimating the Intensity and frequency of the remaining buzz. The process of removing the formants is called inverse filtering, and the remaining signal after the subtraction of the filtered modeled signal is called the reside.

The number which describe the Intensity and frequency of the buzz, the formants, and the reside signal, can be stored or transmitted somewhere else. LPC synthesizes the speech signal by reversing the process: use the buzz parameters and the residue to create a source signal. Use the formants to create a filter (which represents the tube), and run the sources through the filter, resulting in speech.

Because speech signals vary with time, this process is done on short chunks of the speech signal, which are called frames; generally 30 to 50 frames per second give intelligible speech with good compression.

LPC is frequently used for transmitting spectral envelope information, and as such it has to be tolerant of transmission errors. Transmission of the filter coefficients directly is undesirable, since they are very sensitive to errors. In other words, a very small error can distort the whole spectrum, or worse, a small error might make the prediction filter unstable.

LPC is generally used for speech analysis and resynthesis. It is used as a form of voice compression by phone companies, for example in the GSM standard. It is also used for secure wireless, where voice must be digitized, encrypted and sent over a narrow voice channel.

In the LPC analysis one tries to predict ${ }^{x_{n}}$ on the basis of the previous samples,

$x_{n}^{\prime}=\sum a_{k} x_{n-k}$

then $\left\{a_{1}, a_{2}, \ldots \ldots \ldots, a_{p}\right\}$ can be chosen to minimize the prediction power $Q_{p}$ where

$Q_{p}=E\left[\left|x_{n}-x_{n}^{\prime}\right|^{2}\right]$

Linear Predictive Coding is used to extract the LPCC coefficients from the speech tokens. The LPCC coefficients are then converted to cepstral coefficients. The cepstral coefficients are normalized in between 1 and -1 . The speech is blocked into overlapping frames of $20 \mathrm{~ms}$ every 10ms using Hamming window. LPCC was implemented using the autocorrelation method. A drawback of LPCC estimates is their high sensitivity to quantization noise. Convert LPCC coefficients into cepstral coefficients where the cepstral order is the LPCC order and to decrease the sensitivity of high and low-order cepstral coefficients to noise, the obtained cepstral coefficients are then weighted. 16 Linear Predictive Cepstral Coefficients are considered for windowing. Linear Predictive Coding analysis of speech is based on human perception experiments. Sample the signal with $11 \mathrm{kHz}$. Frames are obtained for each utterance of the speaker form Linear Predicitve Cepstral Coefficients. 


\subsubsection{Mel-Frequency Cepstral Coefficients}

Feature extraction consists of computing representations of the speech signal that are robust to acoustic variation but sensitive to linguistic content. The Mel-filter is used to find band filtering in the frequency domain with a bank of filters. The filter functions used are triangular in shape on a curvear frequency scale. The filter function depends on three parameters: the lower frequency, the central frequency and higher frequency. On a Mel scale the distances between the lower and the central frequencies and that of the higher and the central frequencies are equal. The filter functions are

$H(f)=0$ for $f \leq f_{l}$ and $f \geq f_{h}$

$H(f)=\left(f-f_{l}\right) /\left(f_{c}-f_{l}\right)$ for $f_{l} \leq f \leq f_{c}$

$H(f)=\left(f_{h}-f\right) /\left(f_{h}-f_{c}\right)$ for $f_{c} \leq f \leq f_{h}$

Mel - Frequency cepstral coefficients are found from the Discrete Cosine Transform of the

Filter bank spectrum by using the formula given by Davis and Mermelstein[1980].

$\left.c_{i}=\sum_{j=1}^{N} P_{j} \cos (i \pi / N(j-0.5))\right)$,

$\mathrm{Pj}$ denotes the power in $\mathrm{dB}$ in the $\mathrm{jth}$ filter and $\mathrm{N}$ denotes number of samples.

12 Mel- Frequency coefficients are considered for windowing. Mel-Frequency analysis of speech is based on human perception experiments. Sample the signal with $11 \mathrm{kHz}$, apply the sample speech data to the mel-filter and the filtered signal is trained. Frames are obtained for each utterance of the speaker form Mel-Frequency Cepstral Coefficients.

\subsubsection{PITCH}

Pitch, in speech, the relative highness or lowness of a tone as perceived by the ear, which depends on the number of vibrations per second produced by the vocal cords. Pitch is the main acoustic correlate of tone and intonation.

Pitch is the property of voice and is determined by the rate of vibration of the vocal cords. The greater the number of vibrations per second, the higher the Pitch. The rate of vibration, in turn, is determined by the length and thickness of the vocal cords and by the tightening or relaxation of these cords.

The voice control is dependent largely upon emotional control. When human get excited or frightened, unconsciously the muscles around your voice box or larynx are tightened. The resulting tension in the vocal cords, according to the science of sound, produces a greater frequency of vibration and consequently a higher Pitch. It is an indication of lack of mental poise if you habitually speak in a voice Pitched too high. Frames are obtained for each utterance of the speaker form Intensity.

\subsubsection{INTENSITY}

Vocal Intensity, the major vocal attribute, depends primarily on the amplitude of vocal cord vibrations and thus on the pressure of the subglottic airstream. The greater the expiratory effort, the greater the vocal volume. Another component of vocal Intensity is the radiating efficiency of the sound generator and its superimposed resonator. The larynx has been compared to the physical shape of a horn. The Intensity (or energy flow) of a sound wave is the power (in energy/sec) transmitted through an area of $1 \mathrm{~m}^{2}$ oriented perpendicular to (normal to) the propagation direction of the wave. Almost everyone knows that if they move away from a constant sound source, they perceive a decrease in loudness. Consider the following example: 
International Journal of Computer Science \& Information Technology (IJCSIT) Vol 3, No 4, August 2011

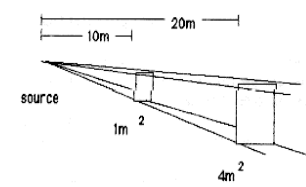

Fig2 Decrease in loudness over distance

Assume that a sound from a source propagates through $1 \mathrm{~m}^{2}$ of air at $10 \mathrm{~m}$ from the source. Looking at the diagram we can see that the power that is concentrated over $1 \mathrm{~m}^{2}$ at $10 \mathrm{~m}$ from the source, is spread over a larger area at the distance of $20 \mathrm{~m}$. The same amount of energy is spread over a larger area, so the Intensity has decreased. Specifically, the area at $20 \mathrm{~m}$ is $4 \mathrm{~m}^{2}$ which is 4 times the area at $10 \mathrm{~m}\left(1 \mathrm{~m}^{2}\right)$, making the energy at $20 \mathrm{~m} 1 / 4$ the Intensity that it was at $10 \mathrm{~m}$. That is:

$$
I=\frac{1}{\gamma^{2}}
$$

where $r$ is the distance from the source.

The sensation of loudness is determined by the Intensity. The greater the Intensity the greater is the perceived loudness. It is usual to symbolise Intensity as I expressed in watt $/ \mathrm{m}^{2}$.

\section{COMPUTER SimulationS}

The self-organizing maps are drawn for features LPCC, MFCC, Pitch and Intensity respectively and the same are shown in Fig.3. It is found that each map is found with different cycle patterns. The weight vectors, shown with the circles, are almost randomly placed. From the Fig.3, it is found that, even after presentation cycles, neighboring neurons, connected by lines, have weight vectors close together.

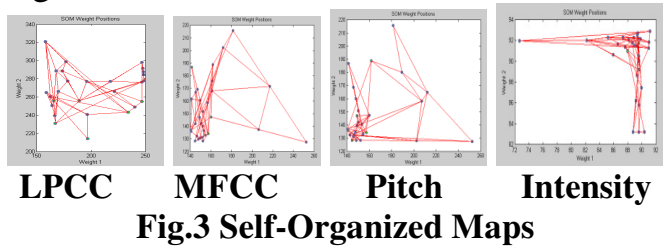

The association of the training data with each of the neurons(cluster centers) for the features LPCC, MFCC, Pitch and Intensity are presented in the Fig.4. The topology is a 10X10 grid with 100 neurons. The maximum number of hits associated with any neuron is 1 . It is found that the number of hits is maximum (12) in the case of MFCC, Pitch and Intensity features.

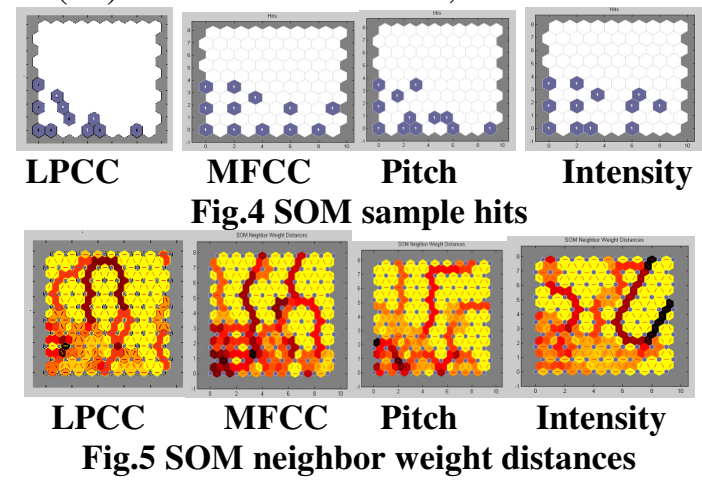


The weight planes for each speaker of the input vector is found against features LPCC, MFCC, Pitch and Intensity. The inputs are assumed to be highly correlated when the connection patterns of inputs were very similar.

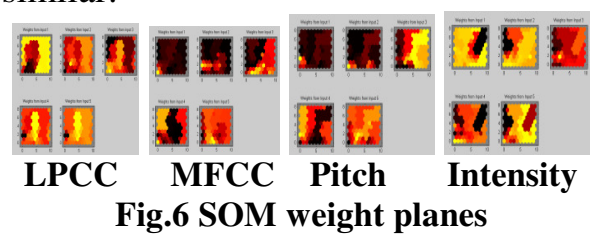

In a bubble chart the data are displayed as a collection of bubbles, each linking the value of the cell in a first selected column to the position of the points on the $X$ (horizontal) axis and the value of the same cell in a second column to the position on the Y (vertical) axis. A bubble chart can highlight various kinds of relationships between data contained in different columns (different dimensions). Clusters can be visually identified as in the Fig.6 and linear or nonlinear relationships between data points can be identified.

\section{Performance}

The set of records available for developing classification methods is generally divided into two disjoint subsets - a training set and a test set. The former is used for deriving the classifier, while the latter is used to measure the accuracy of the classifier. The accuracy of the classifier is determined by the percentage of the test examples that are correctly classified.

There are five speakers and four features. Each speaker is trained and tested with remaining speakers in each case of the features respectively LPCC, MFCC, Pitch and Intensity. Performances are evaluated for each speaker against each feature and presented in the table.

Table 1: Performance of speakers

\begin{tabular}{|c|c|c|c|c|c|c|c|c|}
\hline Feature & \multicolumn{2}{|c|}{ LPCC } & \multicolumn{2}{c|}{ MFCC } & \multicolumn{2}{c|}{ Pitch } & \multicolumn{2}{c|}{ Intensity } \\
\hline $\begin{array}{c}\text { Speaker } \\
\text { number }\end{array}$ & $\begin{array}{c}\text { Performan } \\
\text { ce }(\%)\end{array}$ & $\begin{array}{c}\text { Iteratio } \\
\text { n no }\end{array}$ & $\begin{array}{c}\text { Perfor } \\
\text { mance } \\
(\%)\end{array}$ & $\begin{array}{c}\text { Iterati } \\
\text { on no }\end{array}$ & $\begin{array}{c}\text { Perform } \\
\text { ance } \\
(\%)\end{array}$ & $\begin{array}{c}\text { Iteration } \\
\text { no }\end{array}$ & $\begin{array}{c}\text { Performanc } \\
\text { e } \%)\end{array}$ & $\begin{array}{c}\text { Iteration } \\
\text { no }\end{array}$ \\
\hline Speaker1 & 88.05 & 36 & 89.58 & 1 & 88.56 & 5 & 97.50 & 66 \\
\hline Speaker2 & 84.90 & 34 & 89.19 & 1 & 82.70 & 40 & 97.40 & 24 \\
\hline Speaker3 & 86.04 & 48 & 89.27 & 1 & 98.22 & 1 & 98.54 & 53 \\
\hline Speaker4 & 96.18 & 27 & 89.56 & 1 & 95.34 & 13 & 98.72 & 8 \\
\hline Speaker5 & 93.92 & 23 & 86.58 & 44 & 80.23 & 2 & 98.69 & 8 \\
\hline
\end{tabular}

The performances of each speaker against the features LPCC, MFCC, Pitch and Intensity are depicted in the Fig.7 - 11. All speaker performances except speaker 3 are non- monotonic line graphs where as speaker 3 performance is monotonic one. The speaker 1 performance for Intensity is $90.30 \%$ times that of LPCC, $91.87 \%$ of MFCC and $90.83 \%$ of Pitch. The speaker 2 performance for Intensity is $87.16 \%$ times that of LPCC, $91.57 \%$ of MFCC and $84.90 \%$ of Pitch. The speaker 3 performance for Intensity is $87.31 \%$ times that of LPCC, $90.59 \%$ of MFCC and $99.67 \%$ of Pitch. The speaker 4 performance for Intensity is $97.42 \%$ times that of LPCC, $90.72 \%$ of MFCC and $96.57 \%$ of Pitch. The speaker 5 performance for Intensity is $95.16 \%$ times that of LPCC, $87.72 \%$ of MFCC and $81.29 \%$ of Pitch. 
International Journal of Computer Science \& Information Technology (IJCSIT) Vol 3, No 4, August 2011

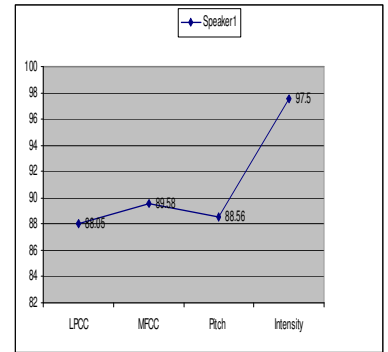

Fig.7 Line graph for speaker1 performance

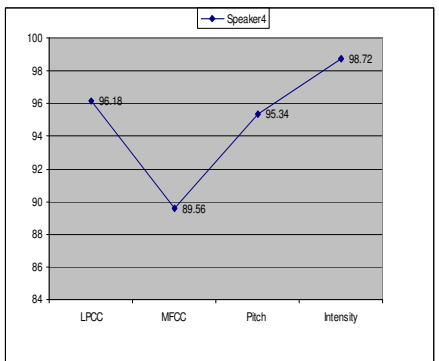

Fig.10 Line graph for speaker4 performance

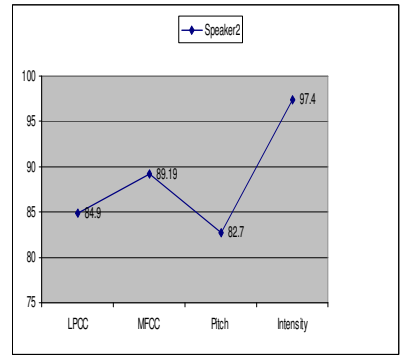

Fig.8 Line graph for speaker2 performance

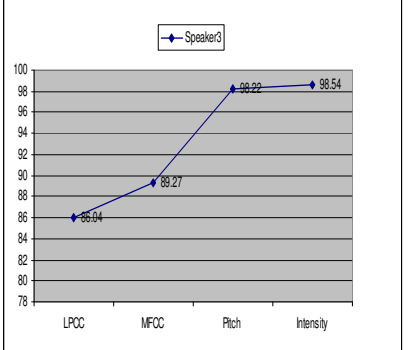

Fig.9 Line graph for speaker3 performance

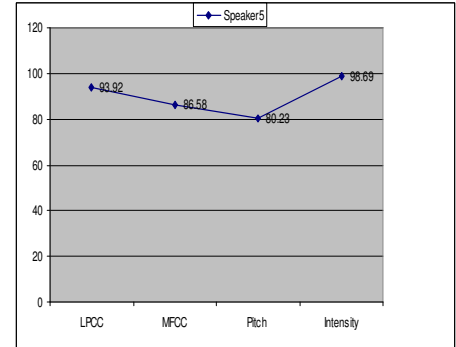

Fig.11 Line graph for speaker5 performance

The mean SOM performance and median SOM performance for each feature is estimated and presented in table.

Table 2: Mean and Median SOM performance

\begin{tabular}{|c|c|c|}
\hline Feature & $\begin{array}{c}\text { Mean- SOM } \\
\text { Performance }\end{array}$ & $\begin{array}{c}\text { Median- } \\
\text { SOM } \\
\text { Performance }\end{array}$ \\
\hline LPCC & 89.81 & 88.05 \\
\hline MFCC & 88.83 & 89.27 \\
\hline Pitch & 89.01 & 88.56 \\
\hline Intensity & 98.17 & 98.54 \\
\hline
\end{tabular}

The Mean SOM performance is compared with Median SOM performance in Fig.12. The Mean SOM performance is decreasing from LPCC to MFCC and increasing from MFCC to Intensity. The Median SOM performance is increasing from LPCC to MFCC and decreasing from MFCC to Pitch and then increasing from Pitch to Intensity. Both Mean and Median SOM performances are steadily increasing from Pitch to Intensity.

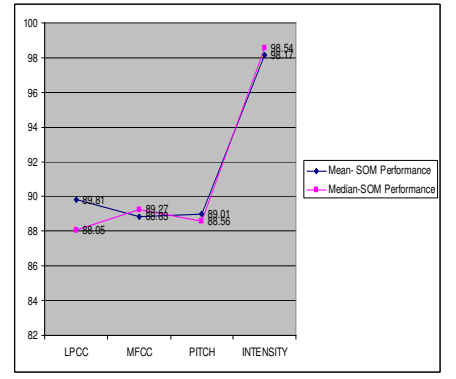

Fig.12 Mean SOM performance Vs Median SOM performances 
The Mean-SOM Performance is a increasing when the speech feature changes from LPCC to MFCC and performance decreases from MFCC to Pitch and the performance rapidly increasing from Pitch to Intensity. The Median - SOM Performance is decreasing from the speech feature LPCC to MFCC and slowly increasing from MFCC to Pitch and rapidly increasing from Pitch to Intensity.

It is found that difference of two central tendency SOM - Performances is least for the Intensity feature.

\section{Analysis of Convergence}

The speech data is trained and tested to find out the analysis of convergence of Kohenen's learning algorithms. The SOM performance of Speakers for feature Intensity is convergent within iteration numbers $66,24,53,8$ and 8 respectively. The convergence is decreasing from Speaker 1 to Speaker 5.

\section{CONCLUSION}

It is found that SOM classifier is apt for the dataset that is chosen to find each speaker performance. In terns of type of classification used, syllable classification has the better performance, having the highest recognition accuracy. This is because scope of vocabulary size becomes smaller when syllable is used as classification unit. In this paper a new approach based on Self-organized maps which is applied to speech recognition is presented. MFCC, LPCC, Pitch and Intensity are taken as cepstral features, these features taken for each speaker for the corresponding 12 words. The obtained frames are trained and tested for SOM. Promising results are obtained. The performance of each speaker varies significantly within the number of iterations. It has shown that SOM classifier has a good rate in both training and testing. It is found that for the speech feature Intensity, SOM speaker performance is best for speakers 1-5 as $99.97 \%, 99.99 \%, 99.96 \%, 99.97 \%$ and $99.69 \%$. It is found that Speaker 2 has attained maximum SOM Performance.

\section{REFERENCES}

[1] Aicha, E.G.Brieuc, C.G.and Fabrice, R.(2004). Self Organizing Map and Symbolic Data. Journal of Symbolic Data Analysis. 2 (1).

[2] A. Sangole, G. K. Knopf, "Geometric representations for high- dimensional data using a spherical SOFM", International Journal of Smart Engineering System Design, vol. 5, pp. 11-20, 2003.

[3] Kohonen, T. (2002). Self-Organizing Neural Networks - Recent Advances and Applications Studies in Fuzziness and Soft Computing. 78: 1-12.

[4] J. Vesanto, E. Alhoniemi, "Clustering of the self-organizing map", IEEE Transactions on Neural Networks, vol. 11, pp. 586-600, 2000.

[5] Kohonen, T.: Self-organising maps. 3rd edn. Springer, Berlin 2000.

[6] Kusumoputro, B. (1999). Development of Self-Organized Network with a Supervised Training in Artificial Odor discrimination System. In Computational Intelligence for Modelling, Control \& Automation. 57-62.

[7] Villmann, T. (1999). Topology Preservation in Self - Organizing Maps, Kohonen Maps, Elsevier. 279-292.

[8] Yamada, T., Hattori, M.,Morisawa, M. and Ito, H. (1999). Sequential Learning for Associative Memory Using Kohonen Feature Map. International Joint Conference on Neural Networks (IJCNN'99). 3: $1920-1923$. 
[9] Zhong, L., Yuanyuan, S. and Runsheng, L. (1999). A Dynamic Neural Network for Syllable Recognition. International Joint Conference on Neural Networks (IJCNN'99). 5: 2997-3001.

[10] G. Deboeck, T. Kohonen, Visual Explorations in Finance with Self-organizing Maps. London: Springer-Verlag, 1998.

[11] K. Haese, "Self-organizing feature maps with self-adjusting learning parameters", IEEE Transactions on Neural Networks, vol. 9, pp. 1270-1278, 1998.

[12] Gavat, I., Valsan, Z. and Sabac, B. (1998). Combining Self-Organizing Map and Multilayer Perceptron in a Neural System for Improved Isolated Word Recognition, Communication 98. 245-255.

[13] TEUVO KOHONEN, SOMERVUO P, Self-Organizing Maps of Symbol Strings with Application to Speech Recognition, (1997).

[14] T. Kohonen. Self-organizing maps of symbol strings. Report A42, Helsinki University of Technology, Laboratory of Computer and Information Science, Espoo, Finland, 1996.

[15] T. Kohonen. Self-Organizing Maps, Springer Series in Information Sciences, vol. 30, Springer, Heidelberg, 1995.

[16] Kohonen, T. (1995). Self - Organizing Maps. Springer, Berlin, Heidelberg.

[17] Frritzke, B.(1994). Growing Cell Structures - A Self- Organizing Network for Unsupervised and Supervised Learning. Neural Networks. 7(9): $1441-1460$.

[18] Hochberg, M.M., Cook, G.D.Renals, S.J.and Robinson, A.J.(1994). Connectionist Model Combination for Large Vocabulary Speech Recognition. IEEE Neural Networks for Signal Processing IV. 269-278.

[19] Berke, L. and Hajela, P.(1991). Application of Neural Nets in structural Optimisation. NATO/AGARD Advanced Study Institute. 23(1-2): 731-745.

[20] Anderson, J. and Rosenfeld, E. (1988). Neurocomputing: Foundations of Research. Cambridge: MIT Press.

[21] TEUVO KOHONEN, Self-Organization and Associative Memory. Springer, Berlin Heidelberg, (1988).

[22] Kohonen, T. (1988b). Self-Organization and Associative Memory. New York: Spring Verlag.

[23] Barto, A.and Anandan, P. (1985). Pattern Recognizing Stochastic Learning Automata. IEEE Transactions on Systems, Man, and Cybernetics. 15: 360-375.

[24] T. Kohonen. Self-Organization and Associative Memory. Springer Series in Information Sciences, vol. 8, Springer, Heidelberg, 1984.

[25] T. Kohonen, "Self-organized formation of topologically correct feature maps", Biological Cybernetics, vol. 43, pp. 59-69, 1982.

\section{BIBLOGRAPHY OF AUTHOR}

Name: Dr.R.L.K.Venkateswarlu Educational Qualifications: M.Tech, Ph.D

Dr.R.L.K.Venkateswarlu has an experience of 20Years. His educational qualifications are M.Tech, Ph.D. Dr.R.L.K.Venkateswarlu has attended and presented good number of research papers in national and

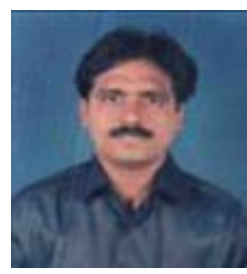
international conferences. He has also national and international publications to his credit. He is gold medalist and awarded best teacher \& researcher from JNTUK \& ISM University. His area of interest is Speech recognition, Pattern recognition, Neural networks \& Earth quake engineering. He is currently working as Professor \& Head in the Department of Information Technology, Sasi Institute of Technology and Engineering, Tadepalligudem. He is life member of Indian Society of Technical Education ISTE, Indian Society of Industrial \& Applied Mathematics and International Journal of Computational Mathematical Ideas. 
Address: Dr.R.L.K.Venkateswarlu

Door No. 12-2-15/1, Seshiahmetta, Rajahmundry - 533104.

Email ID: rlk.maths@gmail.com

Mobile Phone: 9490741799

Land Phone: 0883-2464498

Name: Dr.R.Vasanthakumari.

Educational Qualifications: M.Sc, Ph.D

Dr. R.Vasanthakumari obtained her Ph.D degree from Pondicherry University, in 2005. She is working as a Principal in Government College,

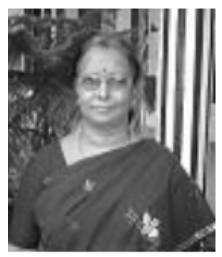
Puducherry, India. She has more then 27 years of teaching experience in P.G. and U.G. colleges. She is guiding many research scholars and has published many papers in national and international conference and in many international journals. Her area of interest is Speech recognition, Pattern recognition, Neural networks \& Fluid Dynamics.

Address: Dr.R.Vasanthakumari.

Principal

Perunthalaivar Kamarajar Arts College,PUDUCHERRY 605107.

Email ID: vasunthara1@gmail.com

Mobile Phone: 09443560868.

Name: A.K.V.Nagayya

Educational Qualifications: MCA

Sri A.K.V.Nagayya working as a lecturer in Department of IT, Sasi Institute of Technology And Engineering. He did his MCA from Andhra

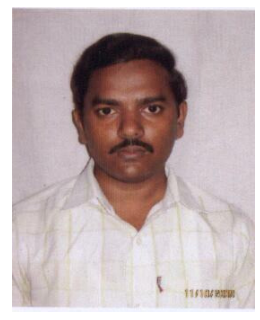

University in 2008. His area of interest is Data Mining \& DataWare Housing and Neural Networks.

Address: A.K.V.Nagayya.

s/o Koti Veera Bhadra rao.

Dr.No: 2-19

Dagguluru

Palakollu Mandal.

W.G.Dist-534250.

Email ID: akvn1983@gmail.com

Mobile Phone: 8985449463 